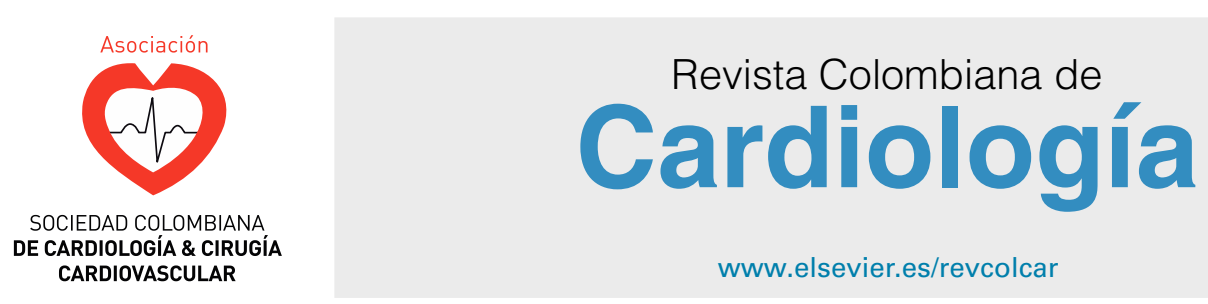

\title{
EDITORIAL
}

\section{¿Qué sugiere el Comité Internacional de Editores de Revistas Médicas de la Sociedad Europea de Cardiología sobre la autoría en artículos científicos?}

\author{
Darío Echeverri
}

\author{
Editor en Jefe, Revista Colombiana de Cardiología
}

Desde hace ya varios años la Revista Colombiana de Cardiología viene estrechando lazos de amistad con la Red de Editores de la Sociedad Europea de Cardiología, lo cual la ubica en una posición privilegiada en Latinoamérica y de cara a otras revistas científicas del país y de la región.

Esta Red está comprometida con el fomento de la implementación de estándares editoriales de alta calidad entre las revistas con interés cardiovascular pertenecientes a las agremiaciones de la Sociedad Europea de Cardiología. Desempeña un papel relevante en la difusión de investigaciones científicas originales y promueve la educación y la armonización de la práctica clínica. En este sentido, la Red de Editores respalda las recomendaciones del Comité Internacional de Editores de Revistas Médicas y actualiza continuamente su documento sobre requisitos uniformes (anteriormente conocido como las directrices de Vancouver) para los manuscritos enviados a revistas biomédicas. Estos incluyen recomendaciones para la conducta, los informes, la edición y la publicación de trabajos académicos. En concordancia con lo anterior, la Revista Colombiana de Cardiología, ya publicó previamente Data Sharing: $A$ New Editorial Initiative of the International Committee of Medical Journal Editors. Implications for the Editors' Network ${ }^{1}$.

Recientemente, la revista Colombiana de Cardiología ha sido invitada a publicar de manera simultánea el documento de Alfonso A, et al. Authorship: From Credit

Véase contenido relacionado en DOI:

https: / / doi.org/10.1016/j.rccar.2019.04.005

Correo electrónico: decheverri@cardioinfantil.org to Accountability. Reflections From the EditorsNietwork. On behalf of the Editors' Network, European Society of Cardiology (ESC) Task Force, revisión con la cual el Comité Internacional de Editores de Revistas Médicas enfatiza que la autoría es un compromiso serio con la responsabilidad de figurar como autor de un artículo científico. Dicho documento hace las recomendaciones para la escogencia de los autores, con base en cuatro requisitos:

1. Contribuciones sustanciales a la concepción o al diseño del trabajo o a la adquisición, análisis o interpretación de datos para el trabajo.

2. Redacción del trabajo o revisión crítica de contenido intelectual importante.

3. Aprobación definitiva de la versión a publicar.

4. Acuerdo para ser responsable de todos los aspectos del trabajo y garantizar que las preguntas relacionadas con la precisión o integridad de cualquier parte del trabajo se investiguen y resuelvan de manera adecuada.

El concepto, muy específico de este manuscrito, es enfatizar la responsabilidad que tiene cada uno de los autores de defender la integridad de todo el trabajo hecho. Cada autor debe comprender el alcance completo del trabajo, saber qué coautores son responsables de contribuciones específicas y tener confianza en la capacidad e integridad de los coautores. Si surgieran preguntas sobre cualquier aspecto de un estudio, la responsabilidad de todos los autores es investigar y garantizar la resolución del problema, que luego se presentará al Editor correspondiente.

Sin duda este relacionamiento de la Revista Colombiana de Cardiología con el Comité Internacional de Editores 
de Revistas Médicas, nos fortalece y el lograr publicar de manera simultánea con más de cincuenta Revistas científicas de Europa este documento, impactará de manera positiva el mejoramiento continuo de nuestros artículos científicos para beneficio de los lectores y de los grupos de investigación.

\section{Referencia}

1. Alfonso F, Adamyan K, Artigou JY, Aschermann M, Boehm M, Buendía A, et al. Data Sharing: A New Editorial Initiative of the International Committee of Medical Journal Editors. Implications for the Editors' Network. Rev Colomb Cardiol. 2017;24:2014-210. 